\title{
Fine-Grained Virtual Network Embedding in 5G Hierarchical Network Infrastructure
}

\author{
Ling-Shu Li, Cai-Xia Liu, Quan Yuan, Xiao-Yang Fang, Yang-Yang Zhang \\ National Digital Switching Center, Zhengzhou, China \\ E-mail: 1lsjdknjr@sjtu.edu.cn
}

\begin{abstract}
Resource allocation and deployment of network Service Function Chain (SFC) is a vital problem of $5 \mathrm{G}$ network virtualization. By introducing SDN and NFV technology, the core network is reclassified as the control layer, forwarding layer and scheduling layer. A Virtual Network Embedding algorithm based on subgraph isomorphism (VNE-MSI) is proposed to allocate resource of each layer in isolated way. The proposed heuristic algorithm collaboratively maps decoupling Service Function Chain with backtracking strategy. Collaborative search strategy is designed according to the characters of virtual Evolved Packet Core. The experimental evaluations show that this method especially performs well in acceptance ratio and revenue-to-cost ratio.
\end{abstract}

Keywords-network virtualization; 5G; virtual network embedding

\section{INTRODUCTION}

Rigid network structure has become a major bottleneck blocking the development of communication networks. In order to achieve flexible resource scheduling in $5 \mathrm{G}$ mobile network, virtual mobile network (VMN) has become a great promising technology[1]. 5G mobile virtual core network is trend to be placed on data center and all network elements in Evolved Packet Core will be controlled by the intelligence Network Orchestrator[2][3].

The Network Orchestrator distributes physical resource from one or several Infrastructure providers (InPs) to generate virtual networks (VN) in an on-demand way. Virtualization makes the core network more flexible and efficient, reduces CAPEX/OPEX and supports more various virtual network function (VNF). Virtual network function depends on virtual network embedding (VNE) technology to select and arrange physical resources[4]. Appropriate VNE algorithm can effectively optimize the resource allocation, improve service quality as well as reduce the energy consumption[5][6].

In this paper we confirm a novel EPC core network virtual structure based on software defined network. In addition, we propose a modified virtual network embedding algorithm based on modified subgraph isomorphism (VNEMSI). VNE-MSI provides two advantages: 1) Flexibility. When the required bandwidths of virtual network flow shoot up suddenly, different to traditional remapping mechanisms, VNE-MSI only need to remap the resources of forwarding layer. 2) Efficiency. Through the decoupling of control layer, forwarding layer and scheduling layer, we are able to achieve fine-grained resource scheduling for better accepting rate and the revenue-to-cost ratio.

\section{RELATED WORK}

Network virtualization has been recognized as a promising solution to the current rigid Internet. Among various virtualization technologies, this paper preliminarily focuses on VNE problem. A set of VNE algorithms have been proposed to ameliorate optimization model and mathematics algorithm. VNE is a NP-hard problem so researchers generally utilize integer linear programming or heuristic algorithm. A heuristic mapping algorithm based on path migration was proposed to handle online $\mathrm{VN}$ requests[7].The algorithm migrates virtual links to different substrate paths to maximize the number of accepted VN requests. But remapping will cause a temporary interruption of service. For virtual network containing a large number of resource constraints, Baumgartner et al. presents a heuristic mapping strategy based on subgraph isomorphism. When a bad mapping decision is detected it can be revised by simply backtracking to the last valid mapping decision, whereas the two stage approach has to remap all links[8]. A dynamic resources allocation algorithm based on periodic characteristics was advocated for the first time to increase the acceptance rate and revenue of InPs[9]. Most studies on the VNE problem in literature focused on resource provisions by reserving the maximum resource demands for a VM throughout its whole lifetime, so most resources were frequently underutilized.

Virtualization in Internet and cloud data center has been studied well in rich literatures[2][8], more and more scholars begin to pay attention to mobile network virtualization. Wireless network virtualization (WNV), with the help of CRAN technology, intends to improve the system performance and user experience[10]. Software defined LTE mobile network was proposed to place the control functions to data center based on operator's mobile network in Finland, so as to make the network more flexible and elastic[11]. In LTE mobile core network, the functions placement problem of two main network element functions, the Serving Gateway (SGW) and PDN Gateway (PGW), was discussed in virtualization environment[12]. As for another EPC network element HSS, a new architecture was proposed for a scalable and elastic HSS through virtualization. Each layer in virtual HSS communicates through standard interfaces[13]. Besides, there are some attempts to transfer the virtualized mobile core network in distributed cloud data centers. A virtual 
mobile network embedding algorithm was proposed to research service chain deployment and topology optimization strategy, with full consideration to the network element restrictions[14]. However, the strategy converges more than 10000 eNodeBs to a single traffic aggregation point (TAP). The high degree of polymerization of service function chain leads to extra expense.

To the author's knowledge, most studies on the VNE problem in literature mainly focused on the Internet. However, different from homogeneous IP points, mobile network elements vary a lot in function properties and resource types. Moreover, there is little hierarchy VNE algorithm available in literature to cope with SDN network. Thus these previous results are less satisfactory for 5G hierarchical framework based on SDN. Mobile Networks Operator is still dearth of well performed strategy to deploy network infrastructure.

In this paper we tentatively propose a hierarchical virtual core network mapping mechanism based on SDN to make further efforts in virtualization. Considering the rapid development of ICT technology, we confirm that 5G network will be deployed in the cloud. Therefore our study focuses on VNE problems in distributed virtual data center. The main contributions of this paper are the following two points: the first is redefining the core network to several layers. The decoupling architecture makes it easier to realize fine-grained resource management and flexible expansion. Then a novel modified graph isomorphism algorithm VNEMSI is proposed to embed virtual networks to physical networks. A modified pruning strategy is proposed to reduce the search space. Results indicate that it especially performs well in acceptance ratio and revenue-to-cost ratio.

\section{HIERARCHICAL VIRTUALIZATION ARCHITECTURE OF MOBILE CORE NETWORK BASED ON SDN}

We assume that the 5G mobile core network comprises the same functionalities and interfaces as the 3GPP LTE Evolved Packet Core (EPC) architecture. EPC is a highly optimized mobile overlay which runs a diverse range of service with guaranteed quality of experience (QoE) and seamless mobility service. Moreover, EPC can capitalize on not only Ethernet-based but also IP-based transport to establish end-to-end connections. Different type of network elements can be deployed in different cloud data center, as illustrated in Fig.1. Despite the similar network functions and elements with traditional architecture, a new SDN-based architecture decouples mobile network control layers from all user plane parts EPC contains data communication network element like SGW and processing network element like Mobile Management Entity (MME). But these elements contain both forwarding function and control function. So in the hierarchical structure of $5 \mathrm{G}$ mobile core network we split the basic elements into two parts. In this way the forwarding plane elements (i.e., the MME-Sw) become simple, stable, and high-performing, while the control plane elements (i.e., the MME-C) can be administrated intensively. Besides, this decouple makes communication network more flexible and easy-orchestrated.

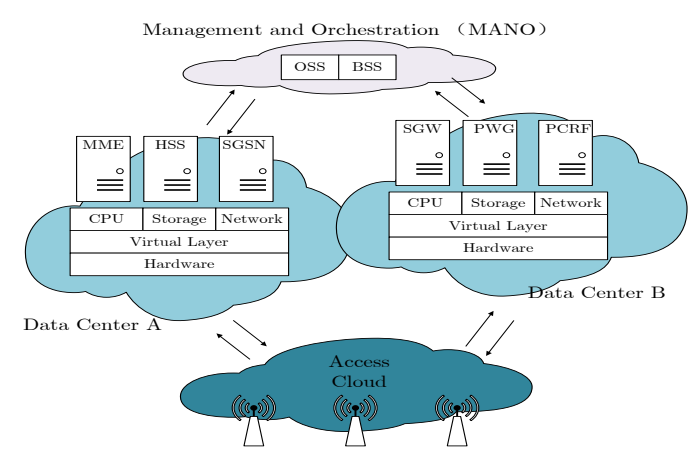

Figure 1. Virtual mobile core network based on cloud data center.

Figure 2 takes HSS for example to illustrate more details about the architecture of virtualized network element. A virtualized HSS can be divided into three layers: virtual resource layer, database control layer and virtual resource management layer. Communication between each layer is through standard interfaces. The average delays when a virtual HSS is used are usually higher than a non-virtualized one, but the difference remains barely observable by endusers[7].As illustrated in Fig.2, Virtual network embedding need overall considerate multiple resources to generate mobile core network. Prime virtual resource includes storage, compute and network resource[9]. The objective and benefit of virtualization is that we can generate services by orchestrating virtual resources on demand. Two typical examples are the Openstack and Cloudstack, which are cloud operating systems that control large pools of abstract resources throughout a datacenter. It's all managed through a dashboard while empowering researchers to provision resources through web interfaces.

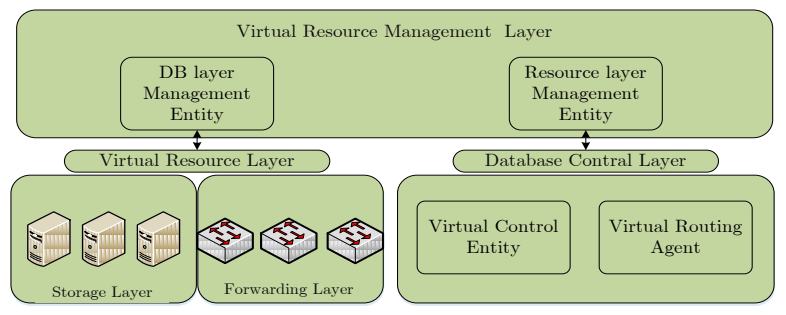

Figure 2. The virtualized HSS architecture.

Similarly, 5G mobile core network is confirmed to be divided as logic control layer, the resource layer and scheduling layer. Therefore, new VNE algorithms are in great demand to cope with the hierarchical structure. Management and scheduling layer is usually pre-mapped in system initialization phase to realize unified resource scheduling. So our VNE algorithm primarily considers about the other two layers.

Virtual nodes of data forwarding layer is similar to virtual switches, which function as multiport bridges. Relatively simple function makes it easier for flexible expansion and shrinkage than traditional elements. According to the EPC communication process, the virtual network requests must be composed of a complete SFC. 
Control layer nodes are orchestrated by Management and Orchestration (MANO) to generate SFC, forming a logical and orderly control surface. Due to the function and deployment of core network element, part of the node mapping has other constraint expect resource limits. For example, P-GW is often used as an anchor to bridge the core network and Public Data Network (PDN) so it has some geographical constraint.

\section{MATHEMATICAL MODEL}

\section{A. Network Module}

A substrate network is represented by a node-and-edge weighted, undirected graph $G^{P}=\left(N^{P}, E^{P}, \eta_{n}^{P}, \eta_{e}^{P}\right)$, where $N^{P}$ and $E^{P}$ are the sets of substrate nodes and links respectively. $n^{P}$ is a substrate node in $N^{P}$ and $e^{P}$ is a substrate link in $E^{P}$. Each $N^{P}$ represents a data center or a set of abstracted physical resource and each $E^{P}$ denotes the communication links (or paths) connecting the substrate nodes. $\eta_{n}^{P}=\left\{\sigma_{n}^{P}, \tau_{n}^{P}, v_{n}^{P}, L o c_{n}^{P}\right\}$ is the set of node property. $\sigma_{n}^{P}$ represents the capacity of computing resource in $N^{P}$, $\tau_{n}^{P}$ represents the capacity of storage resource. $v_{n}^{P}$ is the set of link property. $\operatorname{Loc}_{n}^{P}$ is the geographical constraint. Similarly, a virtual network can also be represented by a undirected graph $G^{V}=\left(N^{V}, E^{V}, \eta_{n}^{V}, \eta_{e}^{V}\right)$, where $N^{V}$ is the set of virtual network functions and $E^{V}$ is the set of virtual links. Each virtual node $\eta_{n}^{V}$ represents a set of virtual machines that host specific applications. Each virtual edge $\eta_{e}^{V}$ represents a communication link between two virtual nodes.

TABLE I. DESCRIPTION OF THE PARAMETERS

\begin{tabular}{|c|c|}
\hline Description & Notation \\
\hline Physical network & $G^{P}=\left(\begin{array}{llll}N^{P}, & E^{P}, & \eta_{n}^{P}, & \eta_{e}^{P}\end{array}\right)$ \\
\hline Virtual network require & $G^{V}=\left(\begin{array}{llll}N^{V}, & E^{V}, & \eta_{n}^{V}, & \eta_{e}^{V}\end{array}\right)$ \\
\hline Set of physical nodes & $N^{P}$ \\
\hline Set of physical links & $E^{P}$ \\
\hline Set of virtual nodes & $N^{V}$ \\
\hline Set of virtual links & $E^{V}$ \\
\hline $\begin{array}{l}\text { Property set of physical } \\
\text { nodes }\end{array}$ & $\eta_{n}^{P}=\left\{\sigma_{n}^{P}, \tau_{n}^{P}, v_{n}^{P}, \operatorname{Loc}_{n}^{P}\right\}$ \\
\hline $\begin{array}{c}\text { Link property of physical } \\
\text { links }\end{array}$ & $v_{n}^{P}$ \\
\hline $\begin{array}{c}\text { Property set of virtual } \\
\text { nodes }\end{array}$ & $\eta_{n}^{V}=\left\{\sigma_{n}^{V}, \tau_{n}^{V}, v_{n}^{V}, L o c_{n}^{V}\right\}$ \\
\hline $\begin{array}{c}\text { Link property of virtual } \\
\text { links }\end{array}$ & $v_{n}^{V}$ \\
\hline Node embedding & $M_{N}: N^{V} \rightarrow N^{P}$ \\
\hline Link embedding & $M_{E}: E^{V} \rightarrow L^{P}$ \\
\hline
\end{tabular}

In details, Virtual network mapping is to find a embedding that makes each virtual node at least map on a physical node, each virtual link map to at least one acyclic physical link. If all the constraints are satisfied, it is referred as a valid mapping. Then the best embedding is selected to maximize special index or cost function.

\section{B. Mapping Strategy Based On Subgraph Isomorphism}

Subgraph isomorphism problem is defined as follows: given a query graph $Q$ and a data graph $\mathrm{G}$, find all embedding of query graph Q in data graph G. Graph algorithms are usually based on the "filter-validation" strategy. Firstly algorithms prune part of the data graph to get candidate data graph based on map index. Then algorithms verify the correctness of candidate figure in the validation phase.

Traditional embedding algorithms map logic control layer nodes in the first step and then map forwarding layer in the second step. Such two stage embedding algorithm often leads to embedding failure due to link bandwidth depleted. Furthermore, it's easy to produce a large number of resource fragmentations because there is no overall management of resources. VNE based on subgraph isomorphism is a one phase algorithm that can effectively reduce the search space and avoid generate over-length path.

\section{Constrained Condition}

Under the single-path embedding case, the remaining Available CPU Resource is defined as

$$
R_{N}\left(n^{P}\right)=c\left(n^{P}\right)-\sum_{n^{V} \mid M_{N}\left(n^{V}\right)=n^{P}} c\left(n^{V}\right)
$$

The remaining available bandwidth resource is defined as

$$
R_{E}\left(e^{P}\right)=b\left(e^{P}\right)-\sum_{e^{V} \mid M_{N}\left(e^{V}\right)=e^{P}} b\left(e^{V}\right)
$$

The mapping must obey resource constraints of the physical nodes and links, given by

$$
\left\{\begin{array}{l}
c\left(n^{V}\right) \leq R_{N}\left(M_{N}\left(\mathrm{n}^{V}\right)\right) \\
b\left(\mathrm{e}^{V}\right) \leq R_{E}\left(M_{E}\left(\mathrm{e}^{V}\right)\right)
\end{array}\right.
$$

\section{Cost and Revenue Models}

The cost and revenue of InPs acquired by embedding virtual networks to the physical networks can be defined differently in diverse economic models. Similar to the revenue models in previous study[15], we define the cost as

$$
C\left(G^{v}\right)=a \sum_{n^{v} \in N^{v}} C\left(n^{v}\right)+(1-a) \sum_{e^{v} \in E^{v}} B\left(e^{v}\right) \bullet \operatorname{length}\left(e^{v}\right)
$$

We presume that the SPs usually sign a long-term contract(e.g., service agreement level) with the InPs. In this contract, the SPs would pay the InPs a fixed amount in the beginning and make additional payment for actual usage. Under this revenue model, for each VNR, its revenue consists of a fixed revenue part and a variable revenue part. 


$$
R\left(G^{v}\right)=R_{V}+R_{f}
$$

The additional payment is usually a "pay-peruse" model, whose corresponding revenue model is assumed as

$$
R_{v}=a \sum_{n^{v} \in N^{v}} C\left(n^{v}\right)+(1-a) \sum_{e^{v} \in E^{v}} B\left(e^{v}\right)
$$

$C\left(n^{v}\right)$ and $B\left(e^{v}\right)$ are the usage of unit computing and bandwidth respectively. The fixed revenue part corresponds to the amortized one from the lump-sum payment of the user to the cloud provider during the contract period, defined as $R_{f}=W_{T} / n_{T} . W_{T}$ is the lump-sum payment for duration $\mathrm{T}$ and $n_{T}$ is the total number of VNRs in the duration.

In addition, the revenue-to-cost ratio $\omega=R\left(G^{v}\right) / C\left(G^{v}\right)$ is often used to measure the pros and cons of a mapping strategy. Efficient online VNE algorithm can maximize the network long-term revenue-to-cost ratio and reduce the additional communication delays.

\section{VIRTUAL NETWORK EMBEDDING ALGORITHM BASED ON MODIFIED SUBGRAPH ISOMORPHISM}

\section{A. Hierarchy Architecture}

5G mobile communication network will be an SDN network. We primary consider about the mapping problem about the logic control layer and the resource layer. While prior VNE algorithms generally confirm the two layers as a whole, in this paper two resource slices are utilized to bear the virtual network cooperatively. Although each layer's elements compose their own topology structure, the control unit and the forwarding unit of the same element have some information interaction. In another word, there are some links between this two parallel layers. So the improved subgraph isomorphism algorithm tries to map them at the same time, and the relevancy of two layers is considered. We allow connections shorter than a predefined distance value $\mathrm{k}$ (in terms of hops), thus a control node has access to more forwarding resources around it.

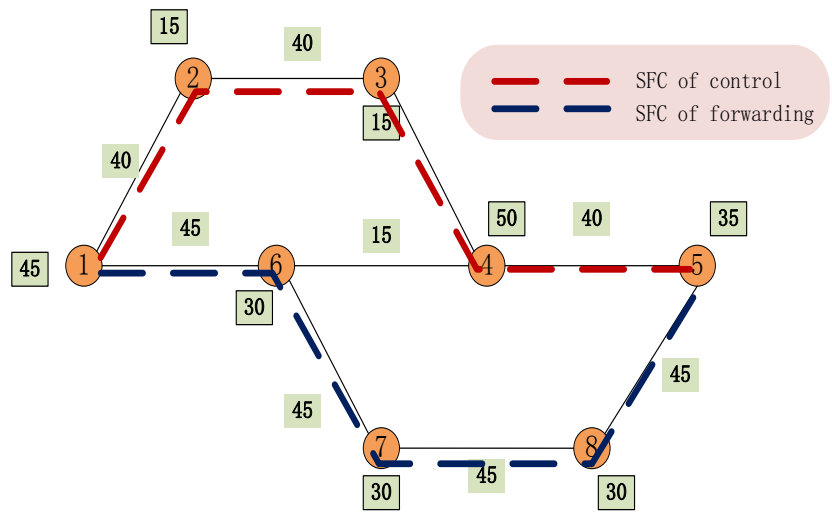

Figure 3. The Hierarchy virtual network mapping model
The establishment of a new SFC can be divided into three steps. First of all, build logic functions chain according to the request of business; then, generate remaining logical view of the underlying network resources. It's based on the usage of accepted VNR resource and the current network status; finally, embed SFC to physical network and output deployment plan.

As shown in figure 3 , the control plane and data plane in traditional mapping method are tightly coupled, which causes resources squander and can't schedule on-demand. To solve above problems, we propose a modified virtual network embedding algorithm based on modified subgraph isomorphism VNE-MSI. VF2 algorithm is a kind of high efficiency mainstream map matching algorithm, but in vEPC environment the traditional VF2 algorithm can neither map multiple VNFs to the same physical node nor allow more jump connections between network nodes. So the topological optimization design can't make full use of substrate resources.

In VNE-MSI model, there are at least one vSwitch (forwarding layer node) and one vHost (control layer node) in a virtual node. The control layer nodes of SFC should be mapped orderly, while the map of forwarding nodes considers more about the capacity of flexible expansion and shrinkage.

\section{B. VNE-MSI Algorithm}

Mainstream graph matching algorithms are based on tree search using backtrack. Heuristic methods are usually utilized to reduce the search space, which is called Prune. Our VNE-MSI is originated from VF2 algorithm, which performed well in graph search. In VNE-MSI the pruning strategy is modified to increase efficiency and the mapping strategy is changed to cope with SDN hierarchy network. Iterative process is similar to depth first search process, which carries out until the current matching state contains all nodes in VNRs. In the process of search, the control layer service chain and forwarding layer service chain alternate map in a coordinate way. Algorithm 1 shows the details of VNE-MSI.

Algorithm 1: $\operatorname{MSI}\left(G^{P}, G^{V}, M(s), G_{s u b}^{V}\right)$

Input: physical network $G^{P}$, virtual network $G^{V}$,

Initial match state $M(s)$, matched graph $G_{\text {sub }}^{V}$;

Output: embedding relationship $M\left(G_{\text {sub }}^{V}\right)$;

1: genpairs $\left(G^{P}, G^{V}, G_{\text {sub }}^{V}\right)$

2: for each $\left(n_{P}, n_{V}\right)$ in $\mathrm{C}$ do

3: if $\operatorname{valid}\left(\mathrm{M}\left(\mathrm{G}_{\mathrm{sub}}^{\mathrm{v}}\right),\left(n_{V}, n_{P}\right), G^{P}\right)$ then

4: update $\mathrm{G}_{\text {sub }}^{\mathrm{V}}$ and $\mathrm{M}\left(\mathrm{G}_{\text {sub }}^{\mathrm{V}}\right)$, add $\left(n_{V}, n_{P}\right)$

5: iteration, $\operatorname{MSI}\left(G^{P}, G^{V}, M(s), G_{\text {sub }}^{V}\right)$

6: end if

7: if $G_{s u b}^{V}=G^{V}$ then

8: return $M\left(G_{s u b}^{V}\right)$

9: end if

10: end for 
The basic workflow of prune is presented using pseudocode in A1gorithm 2 and described in the following. The function generates the candidate matching pairs form the neighbor of current mapped nodes. At the start of the map, the algorithm computes the candidates set $\mathrm{C}$ as the Cartesian product of all nodes. In the iterative process, the algorithm chooses the neighbor of matching point to generate candidate nodes. Traditional VF2 algorithm uses all nodes to calculate the Cartesian product. Considering the various VNF in SFC has certain logical orders, so we only choose the next virtual node to generate matching pairs at every turn. In other words, we choose only one point in $G^{V}$, instead of all points, to generate candidate matching pairs. Because other points will appear in iterative process, so the algorithm still can traverse the entire search space. In this way the number of candidate matching pairs is reduced comparing with conventional VF2 algorithm. The function $\operatorname{Verify}\left(G^{V}, G^{P}, C\right)$ checks whether the new matching pairs satisfy resource constraints and the position constraint. The function $\operatorname{Rank}(C)$ sorts the physical nodes in ascending order according to CPU resources. Then our algorithm uses the MAX-MIN rule to verify new matching pair. It is aimed at choosing the physical node whose remaining resources is closest to VNR.

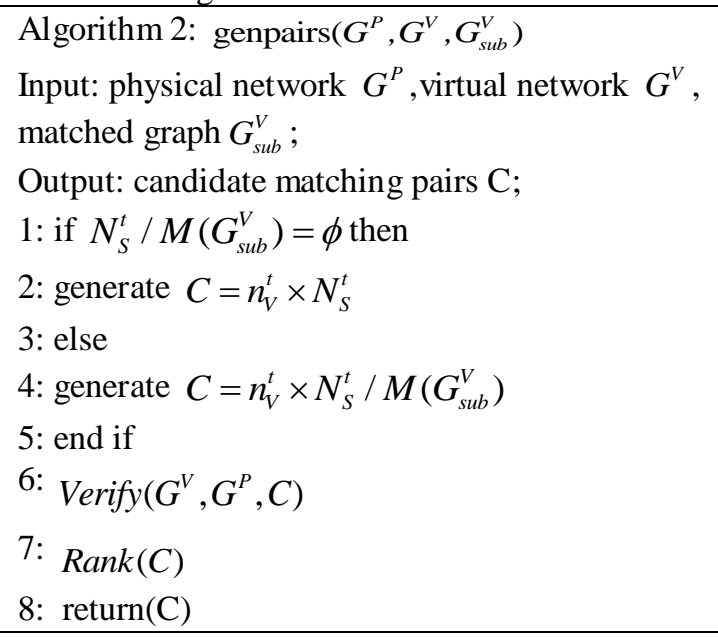

\section{EXPERIMENTAL STUDY}

\section{A. Simulation Settings}

Specifically, we generate $G^{P}$ consists of 50 substrate nodes by the NS-3. And there is an edge between each pair of nodes with a probability of 0.1 , following the similar settings in former work[8]. The computing capacity of each substrate node in $G^{P}$ is randomly drawn from $2000 \mathrm{GHz}$ to $5000 \mathrm{GHz}$, and the bandwidth capacity of each substrate link is set from $10 \mathrm{Mbps}$ to $1000 \mathrm{Mbps}[10]$. The virtual nodes number of each virtual network varies from 2 to 10 , and there is a virtual link between every two virtual nodes with a probability of 0.5 . Assume that the virtual network request $R_{L}$ reach dynamically and obey the Poisson distribution with strength $\lambda \in(400,600)$. The life cycle of virtual network obey negative exponential distribution with $1 / \mu=500$. The virtual network load is expressed as $\lambda / \mu$.

\section{B. Performance Evaluation}

We use acceptance ratio and revenue-to-cost as performance metrics to measure our algorithms. The acceptance ratio measures the percentage of total $\mathrm{VN}$ requests accepted over a given period. The generated revenue and cost (defined in IV-D) are also measured over time. An algorithm can be considered performing better than its counterparts when it generate higher revenue-to-cost ratio in addition to a higher accepting ratio.

Figure 4 illustrates the request acceptance rate of each algorithm. It can be seen from the diagram that VNE-MSI algorithm performs well in request acceptance rate, which shows that the proposed hierarchical mapping method can improve the resource utilization. As you can see in figure 5, the MSI algorithm achieves better long-term average than traditional algorithms. The traditional two phase mapping algorithm often can't embed VNR successfully because of the link bandwidth depleted. Lacking of global planning and resources management, traditional ways are easy to produce a large number of resources pieces. VNE-MSI algorithm conducts overall consideration of node and link resources to achieve load balance, so network utilization is more efficient.

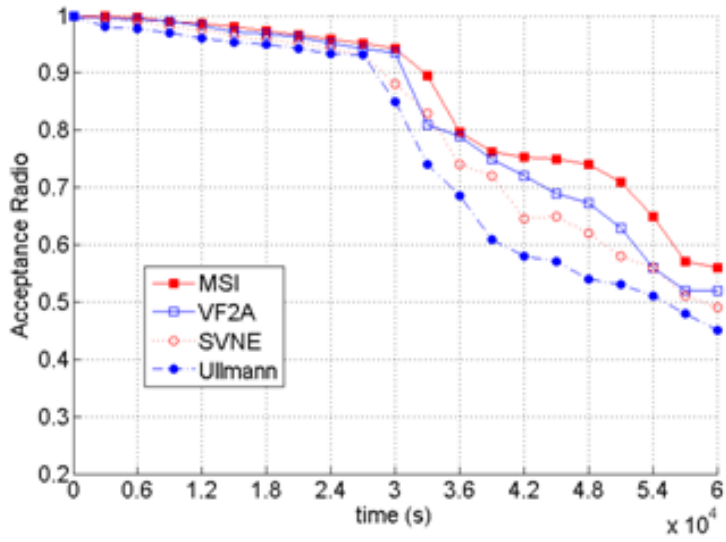

Figure 4. Accumulated acceptance ratio of different algorithm.

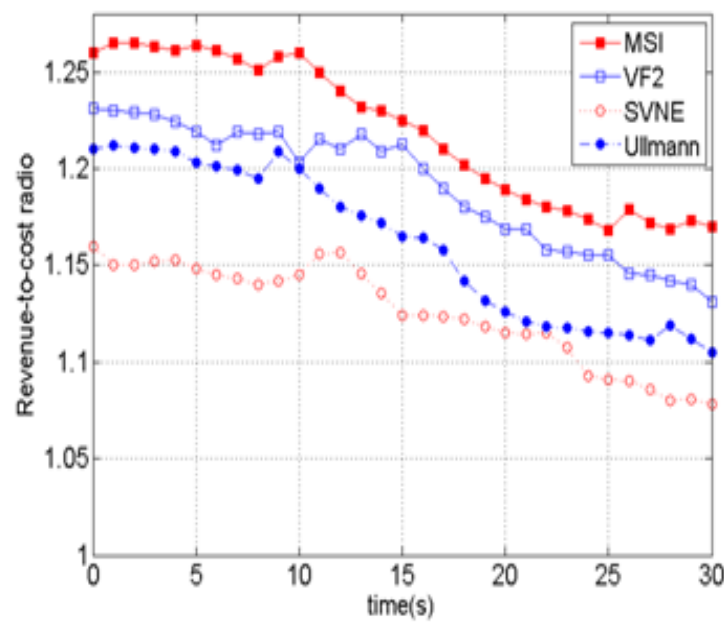

Figure 5. Revenue-to-cost radio under different connectivity rates. 
Figure 6 depicts the impact of node connectivity on mapping quality. We compare the acceptance radio for different predefined node connectivity. The difference is considerably significant, which means more edges in the underlying network will contribute to better acceptance radio based on the sacrifice of computation complexity.

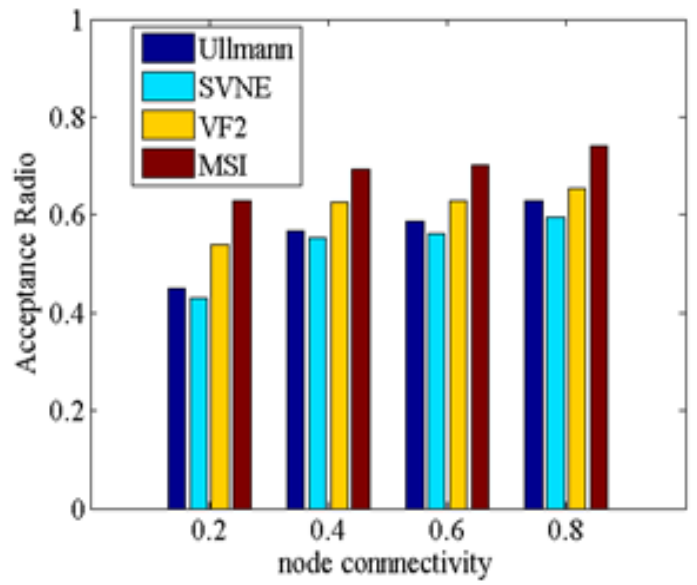

Figure 6. Acceptance radio for different node connectivity

In summary, our proposed hierarchy framework and VNE-MSI algorithm improves the acceptance radio through fine-grained resource utilization, which enables the substrate network to accept more VNRs. Besides, more flexible algorithm also leads to better revenue-to-cost radio.

\section{CONCLUSION}

With the increasing number of terminals and service types, user traffic differentiation is becoming more and more serious. Rigid network structure has become a major bottleneck restricting the development of 5G mobile communication network. Virtual network embedding is a key enabling technology of virtualization. Suitable Virtual network embedding algorithm can optimize the network resource allocation effectively, improve service quality and reduce the energy consumption. This paper mainly studies the virtual function deployment problem. A kind of hierarchical collaborative mapping algorithm VNE-MSI is put forward based on subgraph isomorphism. It makes use of the topological characteristics to take reasonable pruning. Besides, VNE-MSI decouples the control chain and the forwarding layer, which not only achieves fine-grained resource utilization, but also makes the algorithm model sensitive to load changes. Experimental results show that the algorithm can improve the acceptance rate and revenue, realize the optimization of network deployment and enhance the user experience.

\section{REFERENCES}

[1] OSSEIRAN A, BOCCARDI F, BRAUN V, et al. Scenarios for 5G mobile and wireless communications: the vision of the METIS
project[J]. IEEE Communications Magazine, 2014, 52(5): 26-35. doi:10.1109/MCOM.2014.6815890.

[2] BARBAROSSA S, SARDELLITTI S, and DI LORENZO P. Communicating while computing: distributed mobile cloud computing over 5G heterogeneous networks[J]. IEEE Signal Processing Magazine, 2014, 31(6): 45-55. doi: 10.1109 / MSP. 2014. 2334709.

[3] HSU W H, SHIEH Y P. Virtual network mapping algorithm in the cloud infrastructure[J]. Journal of Network and Computer Applications, 2013, 36(6): 1724-1734. doi: 10.1016 / J.JNCA. 2013. 02. 028.

[4] ZHANG N and HAMMAINEN H. Cost efficiency of SDN in LTEbased mobile networks: case Finland[C]. IEEE Networked Systems, Cottbus, Germany, 2015: 1-5. doi:10.1109/NetSys.2015.7089056.

[5] BASTA A, KELLERER W, HOFFMANN M, et al. Applying NFV and SDN to LTE mobile core gateways, the functions placement problem[C]. ACM Workshop on All Things Cellular: Operations, Applications, \& Challenges, Chicago, USA, 2014: 33-38. doi: 10.1145 / 2627585.2627592.

[6] ALIPOUR H, BELQASMI F, ABU-LEBDEH M, et al. Towards HSS as a virtualized service for $5 \mathrm{G}$ networks[C]. IEEE Network and Service Management, Barcelona, Spain, 2015: 153-157. doi: 10.1109 / CNSM. 2015.7367351.

[7] GARG S, DWIVEDI R K, and CHAUHAN H. Efficient utilization of virtual machines in cloud computing using synchronized throttled load balancing $[\mathrm{C}]$. IEEE Next Generation Computing Technologies, Dehradun, India, 2015: 77-80. doi:10.1109/IADCC.2015.7154687.

[8] BAUMGARTNER A, REDDY V S, and BAUSCHERT T. Mobile core network virtualization: a model for combined virtual core network function placement and topology optimization[C]. IEEE Conference on Network Softwarization, London, United Kingdom, 2015: 1-9. doi:10.1109/NETSOFT.2015.7116162.

[9] LISCHKA J, KARL H. A virtual network mapping algorithm based on subgraph isomorphism detection[C]. ACM Workshop on Virtualized Infrastructure Systems and Architectures, Beijing, China, 2009: 81-88. doi:10.1145/1592648.1592662.

[10] FAN Z, CHOI B, XU J, et al. Asymmetric structure-preserving subgraph queries for large graphs[C]. IEEE 31st International Conference on Data Engineering, Seoul, Korea, 2015: 339-350. doi: 10.1109/ICDE.2015.7113296.

[11] ROSA R V, ESTEVE ROTHENBERG C, and MADEIRA E. Virtual data center networks embedding through software defined networking $[\mathrm{C}]$. IEEE Network Operations and Management Symposium, Krakow, Poland, 2014: 1-5. doi: 10.1109 / NOMS. 2014 6838352.

[12] GONZALEZ A, BARRA E, BEGHELLI A, et al. A sub-graph mapping-based algorithm for virtual network allocation over flexible grid networks[C]. IEEE Transparent Optical Networks, Budapest, Hungary, 2015: 1-4. doi:10.1109/ICTON.2015.7193484.

[13] CAO Y, FAN W, and MA S. Virtual Network Mapping: A Graph Pattern Matching Approach[M]. Berlin, Germany, Springer International Publishing, 2015: 49-61. doi:10.1007/978-3-319-204246_6.

[14] HOSSAIN E, HASAN M. 5G cellular: key enabling technologies and research challenges[J]. IEEE Instrumentation \& Measurement Magazine, 2015, 18(3): 11-21. doi:10.1109/MIM.2015.7108393.

[15] GONG L, WEN Y, ZHU Z, et al. Toward profit-seeking virtual network embedding algorithm via global resource capacity[C]. IEEE International Conference on Computer Communications, Toronto, Canada, 2014: 1-9. doi:10.1109/INFOCOM.2014.6847918. 